\title{
Nuclear reprogramming: the strategy used in normal development is also used in somatic cell nuclear transfer and parthenogenesis
}

\author{
Tianlong Gao 1,*, Junke Zheng ${ }^{2, *}$, Fengying Xing, 2,*, Haiyan Fang ${ }^{2}$, Feng Sun ${ }^{1}$, Ayong Yan $^{2}$, Xun Gong ${ }^{2}$, Hui Ding ${ }^{3}$, \\ Fan Tang ${ }^{2}$, Hui Z Sheng ${ }^{2}$ \\ ${ }^{1}$ Program for Graduation Studies, Shanghai Institutes for Biological Sciences, Chinese Academy of Sciences, Shanghai 200031, \\ China; ${ }^{2}$ Center for Developmental Biology, Xinhua Hospital, Shanghai Jiao Tong University, School of Medicine, 1665 Kong Jiang \\ Road, Shanghai 200092, China: ${ }^{3}$ School of Animal Science and Technology, Yangzhou University, Yangzhou 225009, China
}

Somatic cell nuclear transfer (SCNT) and parthenogenesis are alternative forms of reproduction and development, building new life cycles on differentiated somatic cell nuclei and duplicated maternal chromatin, respectively. In the preceding paper (Sun F, et al., Cell Res 2007; 17:117-134.), we showed that an "erase-and-rebuild" strategy is used in normal development to transform the maternal gene expression profile to a zygotic one. Here, we investigate if the same strategy also applies to SCNT and parthenogenesis. The relationship between chromatin and chromatin factors (CFs) during SCNT and parthenogenesis was examined using immunochemical and GFP-fusion protein assays. Results from these studies indicated that soon after nuclear transfer, a majority of CFs dissociated from somatic nuclei and were redistributed to the cytoplasm of the egg. The erasure process in oogenesis is recaptured during the initial phase in SCNT. Most CFs entered pseudo-pronuclei shortly after their formation. In parthenogenesis, all parthenogenotes underwent normal oogenesis, and thus had removed most CFs from chromosomes before the initiation of development. The CFs were subsequently re-associated with female pronuclei in time and sequence similar to that in fertilized embryos. Based on these data, we conclude that the "erase-and-rebuild" process observed in normal development also occurs in SCNT and in parthenogenesis, albeit in altered fashions. The process is responsible for transcription reprogramming in these procedures. The "erase" process in SCNT is compressed and the efficiency is compromised, which likely contribute to the developmental defects often observed in nuclear transfer (nt) embryos. Furthermore, results from this study indicated that the cytoplasm of an egg contains most, if not all, essential components for assembling the zygotic program and can assemble them onto appropriate diploid chromatin of distinct origins.

Cell Research (2007) 17: 135-150. doi: 10.1038/cr.2007.2; published online 6 February 2007

Keywords: nuclear reprogramming, SCNT, parthenogenesis, chromatin factors, chromatin, embryogenesis

\section{Introduction}

It was discovered first in Xenopus and later in many other animal species that once transfered into enucleated eggs, differentiated somatic cells could be reprogrammed

*These three authors contributed equally to this work Correspondence: Hui Z Sheng

Tel: +86-21-55570361; Fax: +86-21-55570017;

E-mail: hzsheng2003@yahoo.com

www.nuclear-reprogramming.com.cn

Received 19 December 2006; revised 10 January 2007; accepted 12 January 2007; published online 6 February 2007 to a totipotent status and to generate live animals [1]. The mechanism underlying the reprogramming process has been the focus of intensive research. Morphological studies indicate that soon after nuclear transfer (nt), the somatic nucleus undergoes a series of changes, which include a massive enlargement in volume, nuclear envelope breakdown, dispersal of nucleoli, premature chromosome condensation, pseudo-pronuclei (PPNs) formation, and nuclear swelling. These morphological changes of the somatic nucleus correlate with and are essential for reprogramming of the gene expression pattern [2-4]. Molecular analyses have revealed that extensive protein exchange between the transplanted nucleus and the cytoplasm of the recipient egg takes place 
after nt [4]. In cloned embryos and animals, X-chromosome inactivation is reversed [5] and telomeres are extended [6]. Patterns in DNA methylation and histone modification are also reprogrammed, although such reprogramming is not efficient [7-12]. Transcription in transplanted nucleus ceases during the process $[4,13]$ and resumes several hours after PPN formation [14]. There is accumulating evidence that reprogramming in transcription profile [15] and epigenetic marks [7-12] is incomplete in the majority of cloned embryos. A large proportion of them display ectopic gene expression and aberrant covalent modifications that are correlated with the donor cell type [7-12, 15]. The primary mechanism responsible for reprogramming the somatic cell gene expression profile to that of the embryo is not yet defined [16-18].

Parthenogenesis is a form of reproduction commonly used in flies, ants, lizards, snakes, fish, birds, reptiles, amphibians, etc.[19]. During the process, the genome of an egg is duplicated and the diploid egg develops without the presence of the male contribution. In mammals, both maternal and paternal genomes are usually required for full development. Parthenogenotes can develop to the mid-gestation stage but not to term in most cases [20-26]. Live mice have been reported to be produced from pathenogenotes, however, through modifications in genomic imprinting [27].

It was hypothesized many decades ago that reprogramming of transplanted somatic nuclei may involve a process relevant to normal development, since transplanted nuclei retrace the normal nuclear events of early development [4, 13]. We examined whether the "erase-and-rebuild" strategy used to reprogram gene expression profile in normal development [41] is also used in somatic cell nuclear transfer (SCNT) and parthenogenesis. It was found in this study that mature eggs retain the ability to dissociate chromatin factor (CFs) from chromatin/nuclei. Shortly after nuclear transplantation, somatic nuclei lose a broad range of CFs. This initial phase in SCNT recaptures the erasure process in oogenesis in an altered fashion. CFs critical for transcription activation and regulation become re-associated with chromatin after PPN formation. During parthenogenesis, a global CF dissociation and re-association occurs as well in a temporal sequence almost identical to that in normal fertilization. Therefore, the "erase-and-rebuild" process occurs also during SCNT and parthenogenesis, and is likely the primary mechanism responsible for transcription reprogramming in these procedures. Evidence from this study also suggests that a compressed duration and compromised efficiency in the erasure process in SCNT contribute to incomplete reprogramming of the epigenetic (including the transcription) program in most nt-embryos. In addition, mature eggs contain most, if not all, essential components for installation of the zygotic developmental program and install it to appropriate diploid chromatin of distinct origins.

\section{Materials and Methods}

\section{Mouse strains}

Adult F1 female mice between DBA and C57 (Beijing, China http://www.vitalriver.com.cn) were used in most SCNT experiments. F1 mice between Kunming white and ICR were also used as an alternative source of eggs. No obvious differences were observed in the reprogramming events initiated by eggs from these two strains.

\section{Parthenogenesis activation of eggs}

Cumulus cell-free eggs were activated in Calcium-free CZB medium [28] containing $5 \mathrm{mM}$ strontium chloride and $5 \mu \mathrm{g} / \mathrm{ml}$ cytochalasin $\mathrm{B}$ (Sigma) at $37^{\circ} \mathrm{C}$ in $5 \% \mathrm{CO}_{2}$. About $1 \mathrm{~h}$ later, the activated eggs were placed in CZB medium containing $5 \mu \mathrm{g} / \mathrm{ml}$ cytochalasin $\mathrm{B}$, and cultured for $5 \mathrm{~h}$. The eggs were then washed several times in fresh CZB medium, and cultured until the zygotes were collected.

\section{Classification of parthenogenesis one-cell embryos}

During parthenogenesis, cytochalasin B prevented the extrusion of the second pole body and both sister chromatids are transformed into pronuclei. The distance between the two pronuclei in the PN1 stage is therefore much less as compared to fertilized PN1 embryos. The parthenogenetic embryos were classified using criteria modified from Adenot et al. [29]. PN1, the pronuclei were newly formed, appearing as two highly condensed dots located at the poles of the spindle, nucleoli may or may not be formed. PN2, the size of pronuclei increased, and all of them contained nucleoli. PN3, the size of pronuclei increased further, the two pronuclei moved towards the center of the embryo and moved closer to each other. PN4, the pronuclei were apposed to each other. PN5, the pronuclei were overlapping each other and in the process of fusion.

\section{Nuclear transfer into enucleated eggs}

The nucleus of the egg was removed by using piezo-assisted micromanipulation (PMAS-CT150, Prime Tech Ltd) in HEPES-buffered CZB medium containing $5 \mu \mathrm{g} / \mathrm{ml}$ cytochalasin $\mathrm{B}$. The remainder of the procedures was the same as those of nuclear transfer into intact eggs described in the accompanying paper.

Quantitative analyses of the relationship between chromatin and GFP-CFs during SCNT using confocal microscope

To obtain the average value representing the starting fluorescent intensity before reprogramming, NIH3 T3 cells expressing weak fluorescent signals were selected and transplanted into enucleated eggs. Nuclear fluorescent levels in 30 transplanted nuclei were measured immediately after $\mathrm{nt}$ (within $5 \mathrm{~s}$ ). The average fluorescent level was used as the starting nuclear fluorescent intensity.

The nuclear fluorescent intensity at different time points after nt was calculated using the following equation: (the nuclear fluorescent intensity/the starting nuclear fluorescent intensity) $\times 100$.

\section{Results}

During SCNT, CFs are first dissociated from the trans- 
A
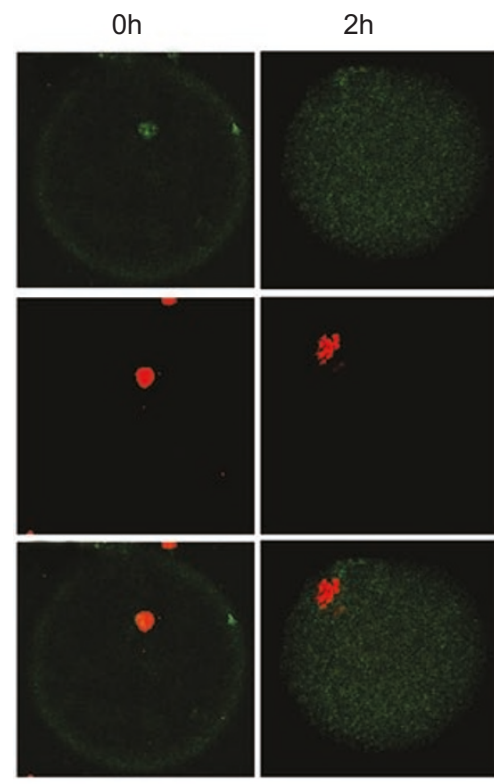

B

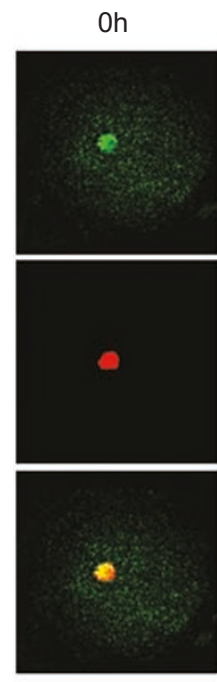

$2 \mathrm{~h}$

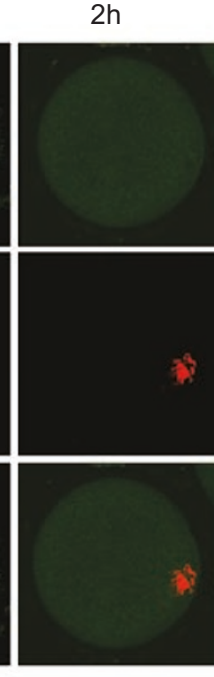

A1h

A4h
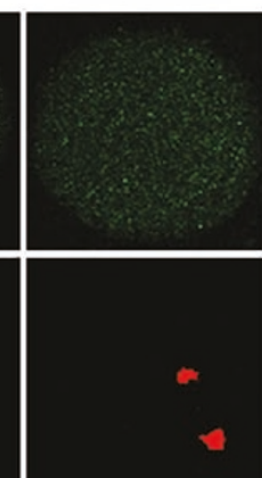

굴

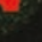

A1h

\begin{abstract}
A1 $h^{*}$
\end{abstract}
A4h

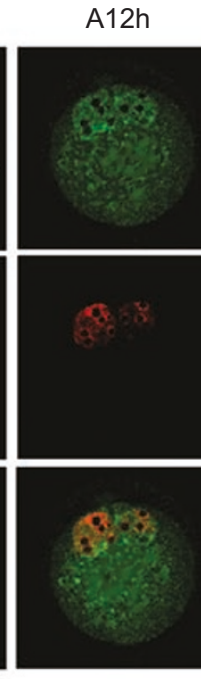

2-cell

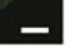

.

C

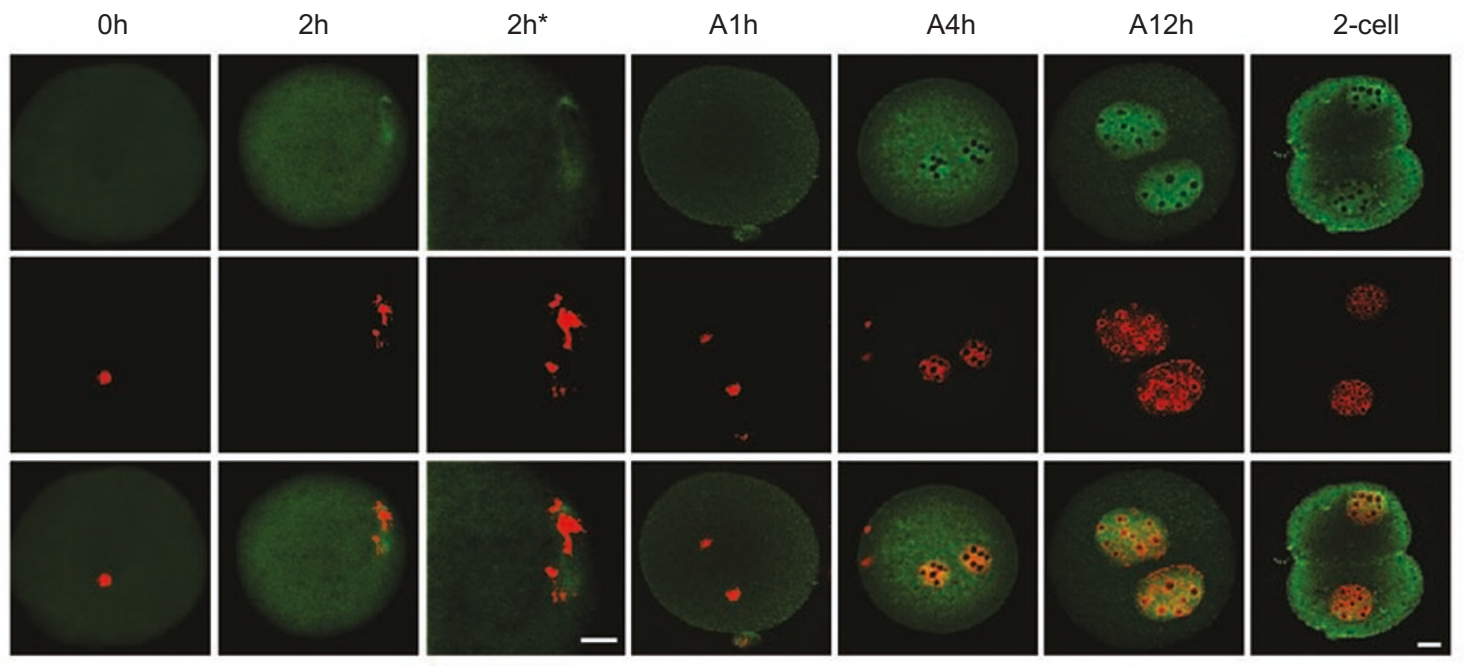


D

Oh

$2 \mathrm{~h}$

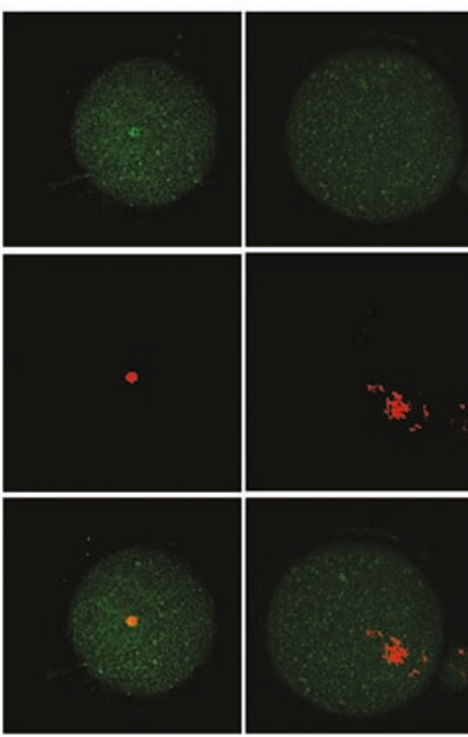

$E$

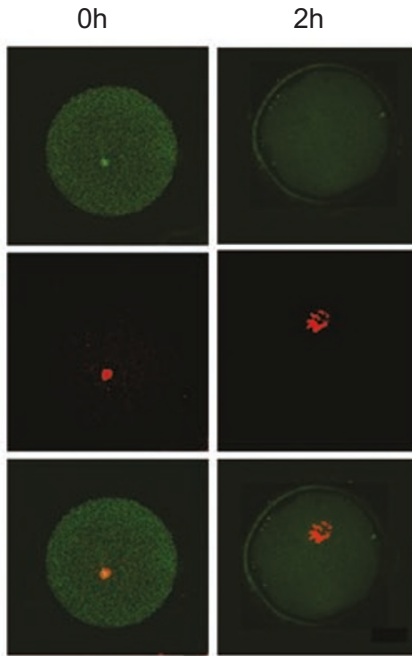

Oh

$2 \mathrm{~h}$
$\mathrm{A} 1 \mathrm{~h}$

A4h

2-cell

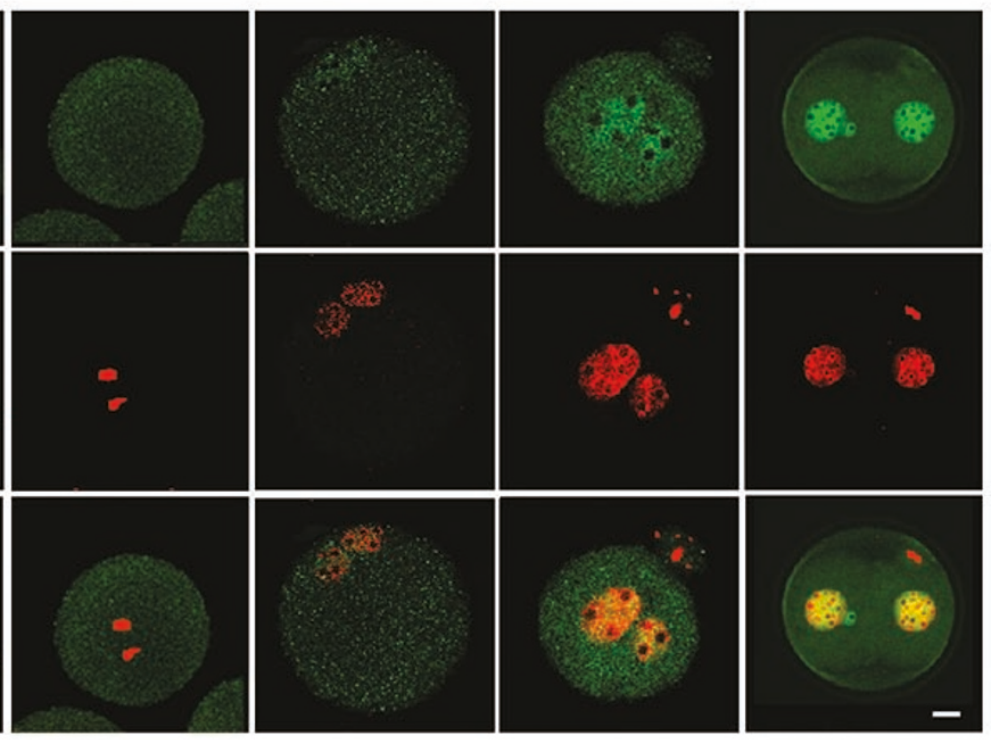

A1h


F

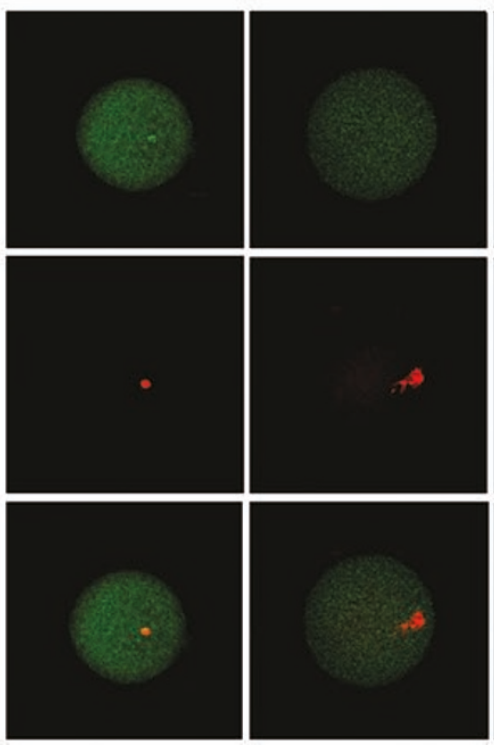

A1h

A4h

$\mathrm{A} 12 \mathrm{~h}$

2-cell

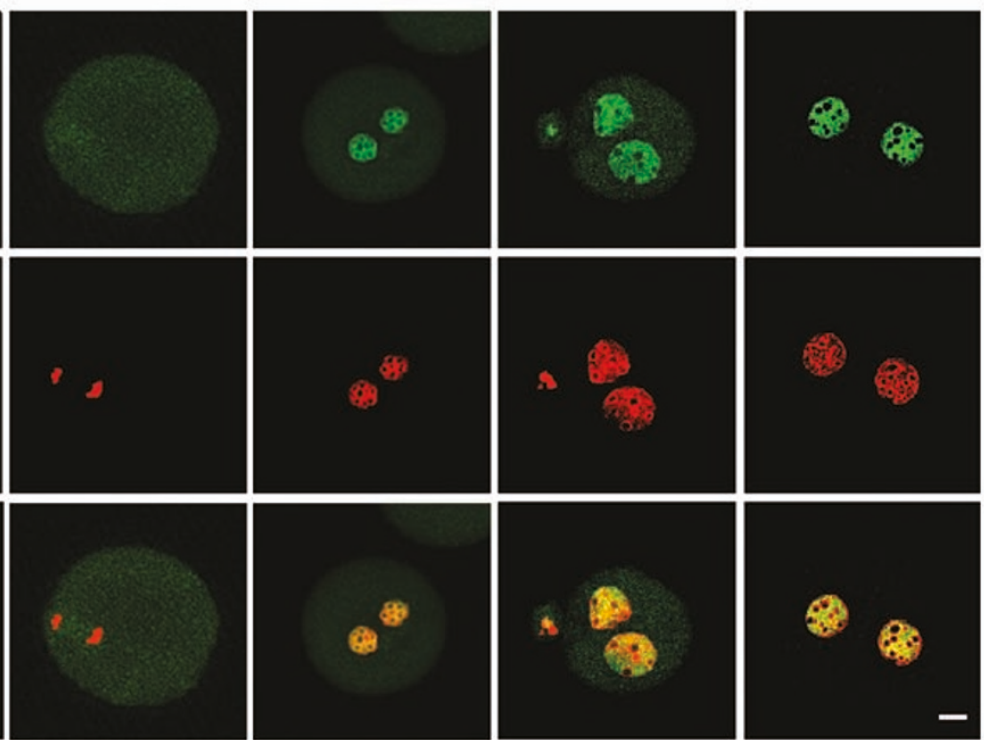


G

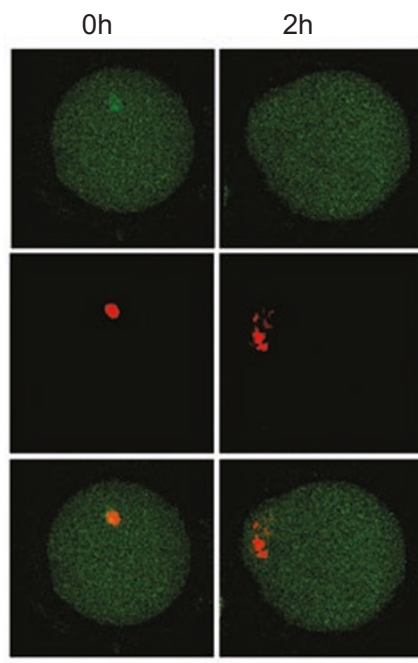

$\mathrm{H}$

Oh
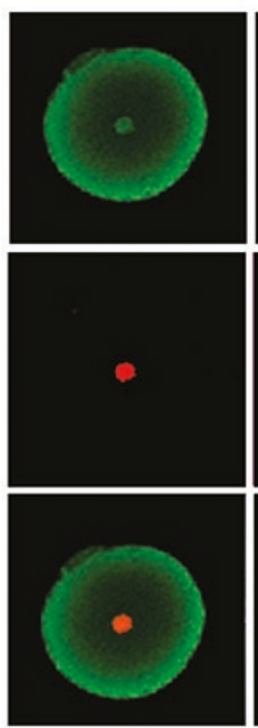

A1h

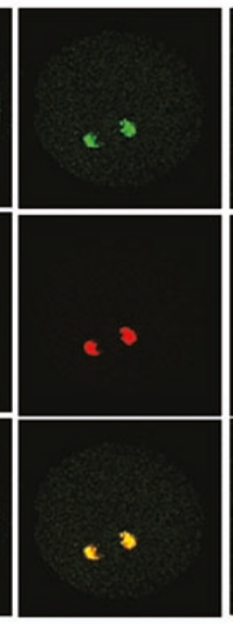

$2 h$
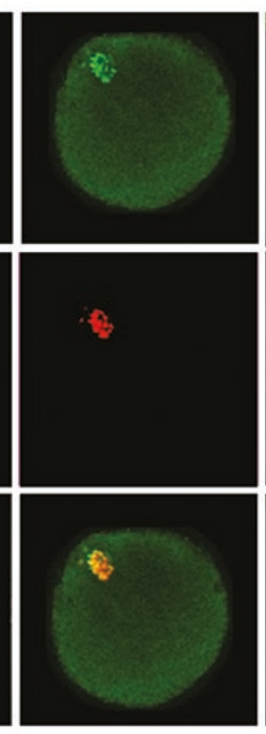

A1h
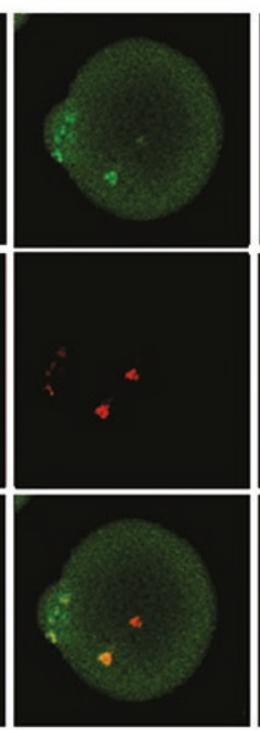

A1h*

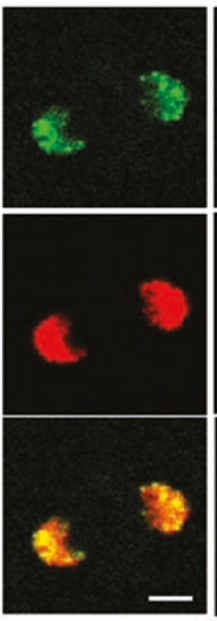

A4h
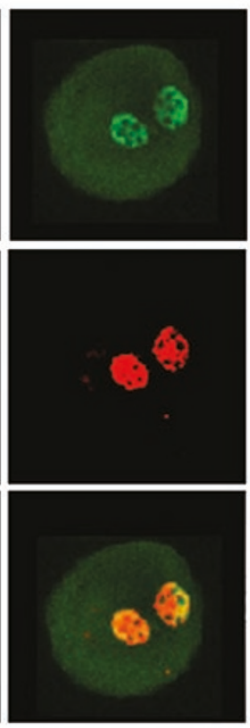

A4h
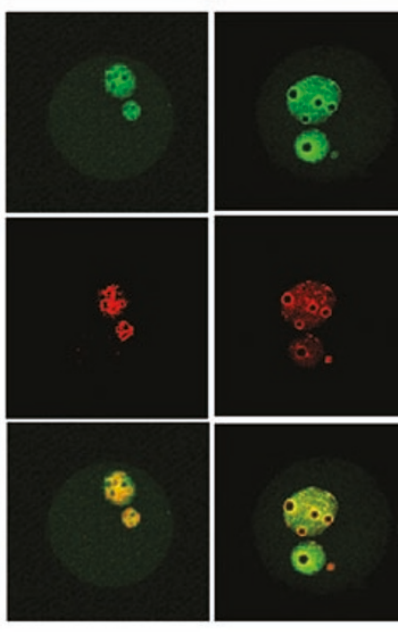

$\mathrm{A} 12 \mathrm{~h}$
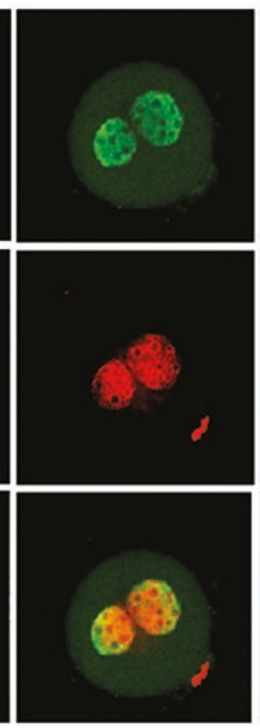

2-cell

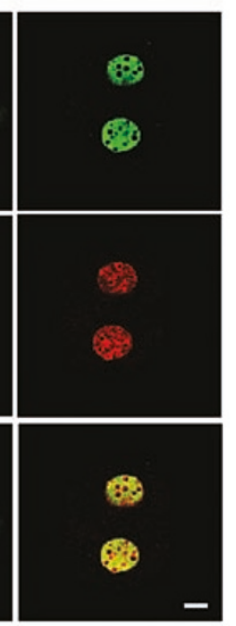

2-cell
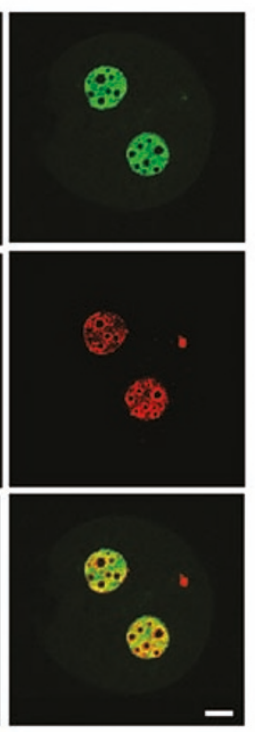

Figure 1 Dynamic changes in nucleus-CF relationship in nt-embryos as revealed by indirect immunofluorescent assays. TBP (A), TAF1 (B), TFIIB (C), BRG1 (D), SRG3 (E), HDAC2 (F), HP1 $\beta$ (G), and AcH4 (H) (green) were detected in the somatic nucleus (red) before nt (not shown) and most of them remained positive shortly after nt ( $<15$ min). However, TBP, TAF1, TFIIB, BRG1, SRG3, HDAC2, and HP1 $\beta$ were no longer detected on chromatin at $2 \mathrm{~h}$ after $\mathrm{nt}(2 \mathrm{~h})$. HP1 $\beta$ became re-associated with chromosomes $1 \mathrm{~h}$ after activation (A1h), and TBP, TAF1, TFIIB, BRG1, SRG3, HDAC2, after pronuclear formation (A4h, A12h, 2-cell). AcH4 remained bound on chromatin/nuclei throughout the course. * indicates a high magnification image of the left. Bar $=10$ and $5 \mu \mathrm{m}$ in low and high (*) magnitude, respectively. See Supplementary information I for more details with these CFs.

planted nucleus and subsequently enter PPNs in a spatiotemporal manner similar to that in normal development

To examine the relationship between somatic nuclei and CFs, freshly isolated mouse cumulus cells were transplanted to enucleated MII eggs. Reconstructed embryos were pre-incubated for $2 \mathrm{~h}$ to enhance reprogramming according to established methods $[30,31]$, and chemically activated
1.5 to $2 \mathrm{~h}$ later. Subcellular distributions of $20 \mathrm{CFs}$ (TBP, TRF3, TAF1, TAF4, TFIIA, TFIIB, Pol II, BRF1, BRG1, SRG3, INI1, YY1, HDAC1, HDAC2, MeCP2, HP1 $\alpha$, HP1 $\beta$, TOPOII $\alpha$, TOPOII $\beta$, and AcH4) in nt-embryos were then examined. As revealed by indirect immunofluorescent assay, all $20 \mathrm{CFs}$ were present in the somatic nuclei before nt (data not shown), and most of them were still detect- 
able shortly after nt ( $<15$ min, Figure 1$)$. Approximately $2 \mathrm{~h}$ after nt, most nuclei went through premature chromosome condensation and entered the anaphase. We detected HP $1 \alpha$, TOPOII $\beta$ and AcH 4 on chromosomes, but not TBP, TRF3, TAF1, TAF4, TFIIA, TFIIB, Pol II, BRF1, BRG1, SRG3, INI1, YY1, HDAC1, HDAC2, MeCP2, HP1 $\beta$, and TOPOII $\alpha$ (Figure 1, Supplementary information I, and Table 1). TRF3, TAF1, TFIIB, BRF1, SRG3, TOPOII $\alpha$, TOPOII $\beta$ were also seen to be associated with structures in the vicinity of chromosomes. These results show that at this stage most transcription factors and regulators become dissociated from the nuclei/chromatin.

Approximately $4 \mathrm{~h}$ after activation, PPNs were formed in most nt-embryos. All 20 CFs, except TRF3, were detected in PPNs. TRF3 was not detected in pronuclei in one-cell embryos but became positive in nuclei at the two-cell stage. By the time when transcription was detected in all reprogrammed nuclei, e.g. $12 \mathrm{~h}$ after activation [14], most $\mathrm{CFs}$ examined were present in the nuclei at high levels (Figure 1, Supplementary information I, and Table 1).

Our data from immunochemical studies indicated that somatic nuclei lose a substantial amount of CFs critical for transcription and other chromatin functions within a few hours after nt and regained them shortly after pronuclear formation. Re-association between PPNs and CFs occurred in a manner similar to that in fertilized embryos $[32-34,41]$.

Exogenous GFP-CF fusion proteins behave in the same way as endogenous proteins during SCNT

To confirm the results from antibody staining, we transplanted individual NIH3T3 cells expressing one of the seven GFP fusion proteins, TBP, TRF3, BRF1, TFIIB, HP1 $\beta, \mathrm{MeCP} 2$, and $\mathrm{H} 2 \mathrm{~B}$, into enucleated eggs. The movement of GFP-CFs and their relationship with chromatin were recorded at a series of time points using the confocal microscope. nt-embryos whose nuclei underwent morphological changes were assigned to the reprogrammed group, and those that did not were placed in the failed-to-reprogram group. As illustrated in Figure 2, GFP-TBP began to dissociate from transplanted nuclei within minutes after nt.

Table 1 A summary in subcellular distribution of CFs in nuclear transfer one-cell embryos

\begin{tabular}{|c|c|c|c|c|c|c|}
\hline & $<15 \mathrm{~min}$ & $2 \mathrm{~h}$ & A1h & A4h & $\mathrm{A} 12 \mathrm{~h}$ & 2 cell \\
\hline TBP & $++^{1} 16^{3}$ & -12 & -21 & $+22 /-1$ & +12 & +9 \\
\hline $\mathrm{TRF}^{2}$ & +11 & -12 & -14 & -14 & -39 & +16 \\
\hline TAF4 & $+9 /-4$ & -10 & -22 & $+16 /-4$ & +11 & +8 \\
\hline TFIIA & -42 & -11 & -20 & $+20 /-2$ & +21 & +9 \\
\hline Pol II & +14 & -22 & -29 & $+23 /-2$ & +11 & +10 \\
\hline BRG1 & +9 & -10 & -22 & +13 & +14 & +11 \\
\hline $\mathrm{SRG}^{2}$ & +12 & -9 & -22 & +19 & +15 & +11 \\
\hline Inil & $+10 /-5$ & -10 & -24 & $+8 /-1$ & $+23 /-10$ & +10 \\
\hline MECP2 & $+9 /-2$ & -13 & -30 & $+14 /-13$ & +14 & +11 \\
\hline HP1 $1 \alpha$ & +17 & $+15 /-4$ & +10 & +17 & +19 & +9 \\
\hline HP $1 \beta$ & +18 & -23 & +10 & +36 & +30 & +15 \\
\hline TOPII $\alpha^{2}$ & $+1 /-7$ & +10 & +13 & $+20 /-4$ & $+18 /-10$ & $+16 /-5$ \\
\hline TOPII $\beta^{2}$ & +11 & -11 & -18 & $+21 /-1$ & +12 & +10 \\
\hline Ac-H4 & +13 & +11 & +11 & +12 & +15 & +7 \\
\hline
\end{tabular}

${ }^{1}+$ and - : signal was or was not detected on chromosomes or PNs, respectively.

${ }^{2}$ Signal is also on structures in the vicinity of chromosomes.

${ }^{3}$ The numbers of embryos with the phenotype (all embryos examined are included in the table, i.e., when only one number is in the column, $100 \%$ of the embryos had the particular phenotype). 

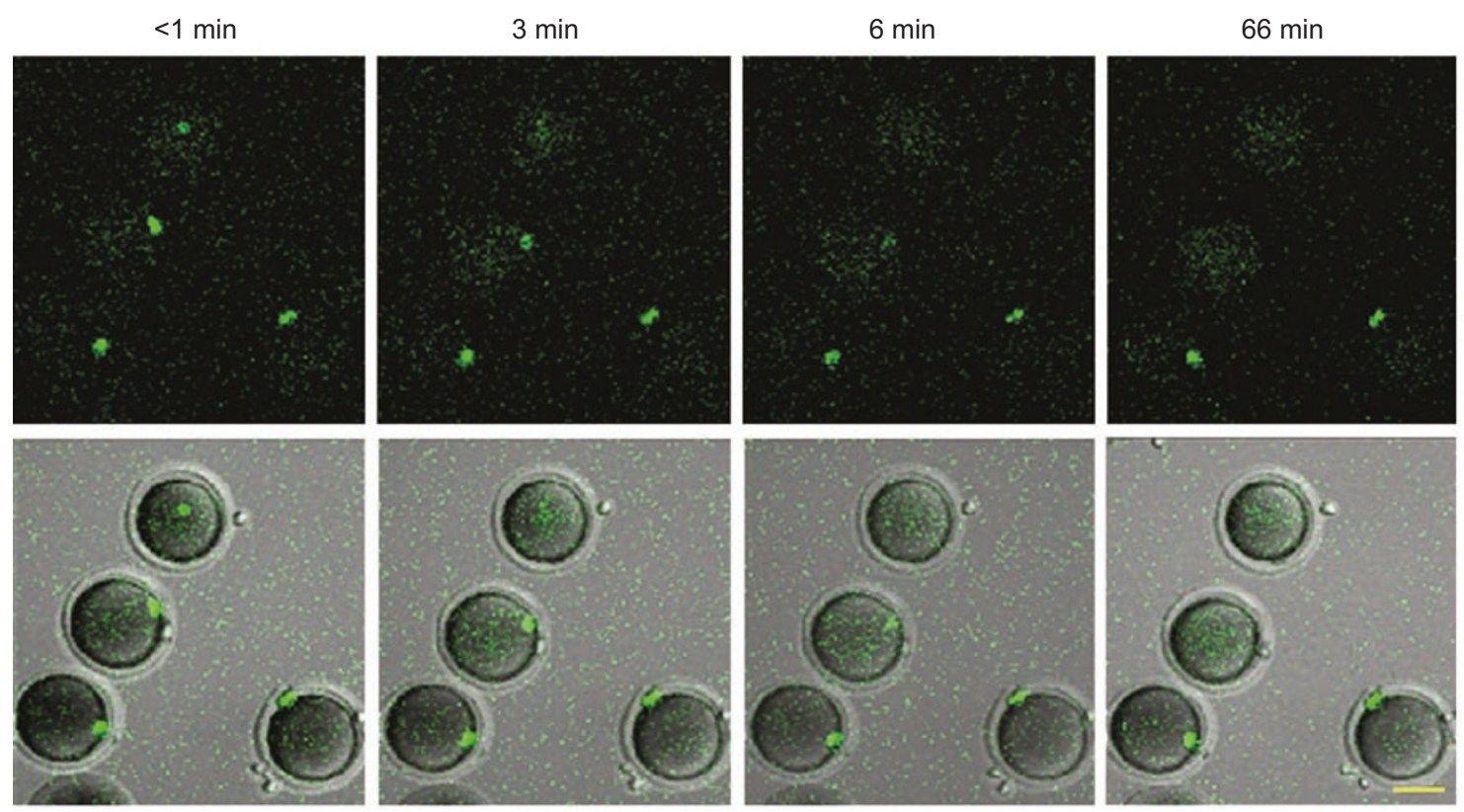

Figure 2 Dissociation of GFP-TBP from somatic nuclei shortly after nt. Immediately $(<1 \mathrm{~min})$ after transplantation of somatic cells into enucleated eggs, all four reconstructed embryos contained a high level of GFP-TBP (green fluorescence) in the nuclei. The level of GFP-TBP decreased gradually in the two embryos located at the top and middle of the image, but not in the two embryos at the bottom $(3,6$, and $66 \mathrm{~min})$. Note that GFP-TBP dissociated from nuclei in different speeds. The fluorescence intensity decreased much faster from the nucleus at the top than from the one at the middle. 1st row, dark-field images. 2nd row, overlaid images of dark and bright fields. $\mathrm{Bar}=50 \mu \mathrm{m}$.

Images and graphs in Figure 3 and Supplementary information II demonstrated that the intensity of GFP-TBP, GFP-TRF3, GFP-TFIIB, GFP-BRF1 and GFP-HP1 $\beta$ in transplanted nuclei dropped significantly to low levels within a few hours after nt. The fluorescence re-appeared in PPNs soon after their formation. These changes did not occur in the failed-to-reprogram group. Quantitative analyses showed that a majority of nuclei in both the reprogrammed and failed-to-reprogram groups lost a substantial amount of GFP-TBP, GFP-TRF3, GFP-TFIIB, GFP-BRF1, and GFP-HP1 $\beta$ after nt. However, the amount of GFP-TBP, GFP-TRF3, GFP-TFIIB, GFP-BRF1, and GFP-HP1 $\beta$ lost by nuclei in the reprogrammed group was significantly greater than in the failed-to-reprogrammed group. In contrast, the amount of GFP-MeCP2 and GFP-H2B lost by both the reprogrammed and failed-to-reprogram groups was not significantly different (Figure 3 and Supplementary information II). Thus, dissociation of GFP-TBP, GFP-TRF3, GFP-TFIIB, GFP-BRF1, and GFP-HP1 $\beta$ is inefficient in the failed-to-reprogram group and is significantly more efficient in the reprogrammed group. These data suggest that phyical changes in transplanted nuclei, e.g. enlargement of the nuclei and nuclear membrane breakdown, etc. maybe required for efficient CF dissociation. CF dissociation is essential for erasure of the existing transcription program from the somatic nuclei and subsequent installation of the zygotic transcription program. The observations that $\mathrm{H} 2 \mathrm{~B}$ and $\mathrm{MeCP} 2$ were retained on chromatin, while many other CFs were dissociated from the somatic nuclei, were consistent with results from biochemical analyses [35]. They were also consistent with results from the immunochemical study described above except for MeCP2 (see below).

Quantitative analyses showed that GFP-CF dissociation occurred soon after nt. Within 15 to 20 min after fusion, the nuclear fluorescent intensity had already decreased in a substantial proportion (46 out of a total 60) of nt-embryos in the reprogrammed groups for TBP, TRF3, TFIIB, BRF1, and HP1 $\beta$. It reached the lowest intensity within either $2 \mathrm{~h}$ after $n t$ or $2 \mathrm{~h}$ after activation in most embryos (55/60). These results indicated that CF dissociation took place actively during the pre-incubation time and extended to $2 \mathrm{~h}$ after activation. The percentage of nuclear GFP-CFs lost after nt was calculated using the average intensity in the control group as $100 \%$. Of the 60 embryos analyzed, nine embryos (15\%) lost $>99 \%, 30$ embryos (50\%) lost between $91 \%$ and $99 \%, 11$ embryos (18.3\%) lost between $81 \%$ and $90 \%$, six embryos (10\%) lost between $61 \%$ and $80 \%$, and four embryos $(6.7 \%)$ lost $\leq 60 \%$ of the GFP-CFs. These 
A

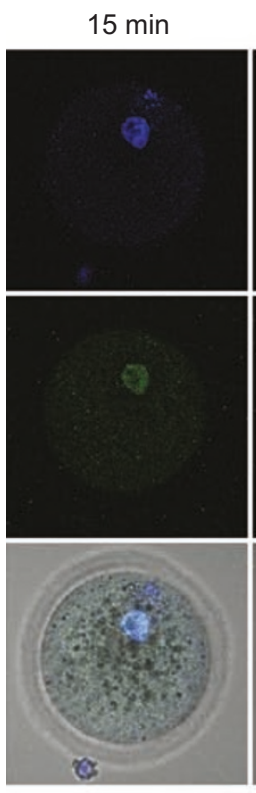

A-2

B
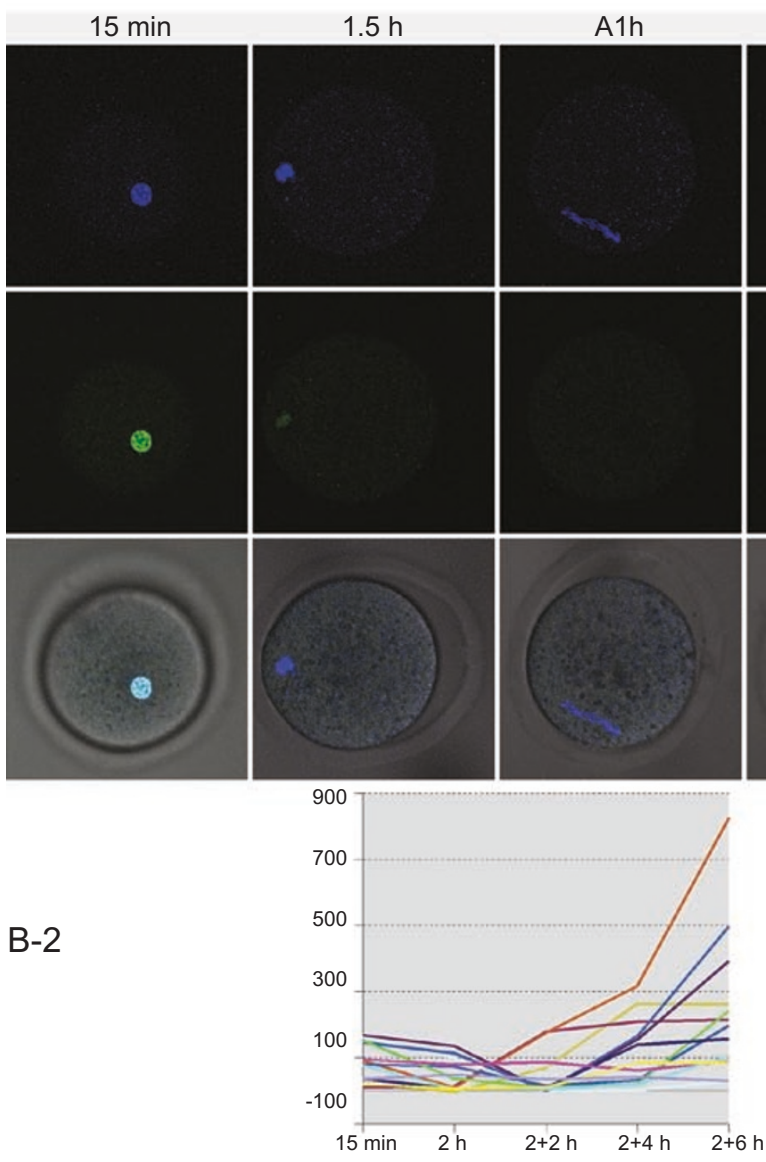

$1.5 \mathrm{~h}$

A1h
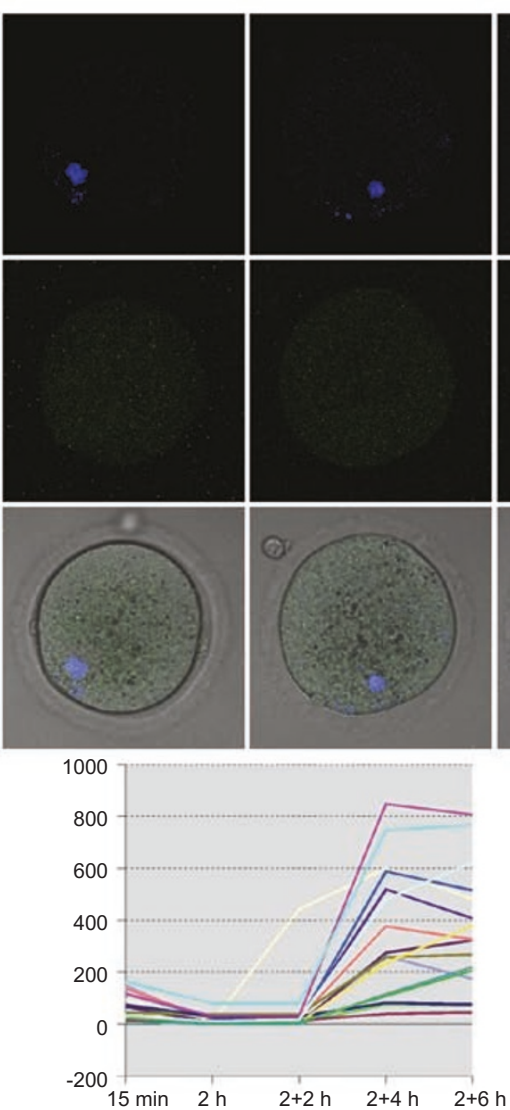

$\mathrm{A} 2 \mathrm{~h}$


$\mathrm{A} 2 \mathrm{~h}$

A4h
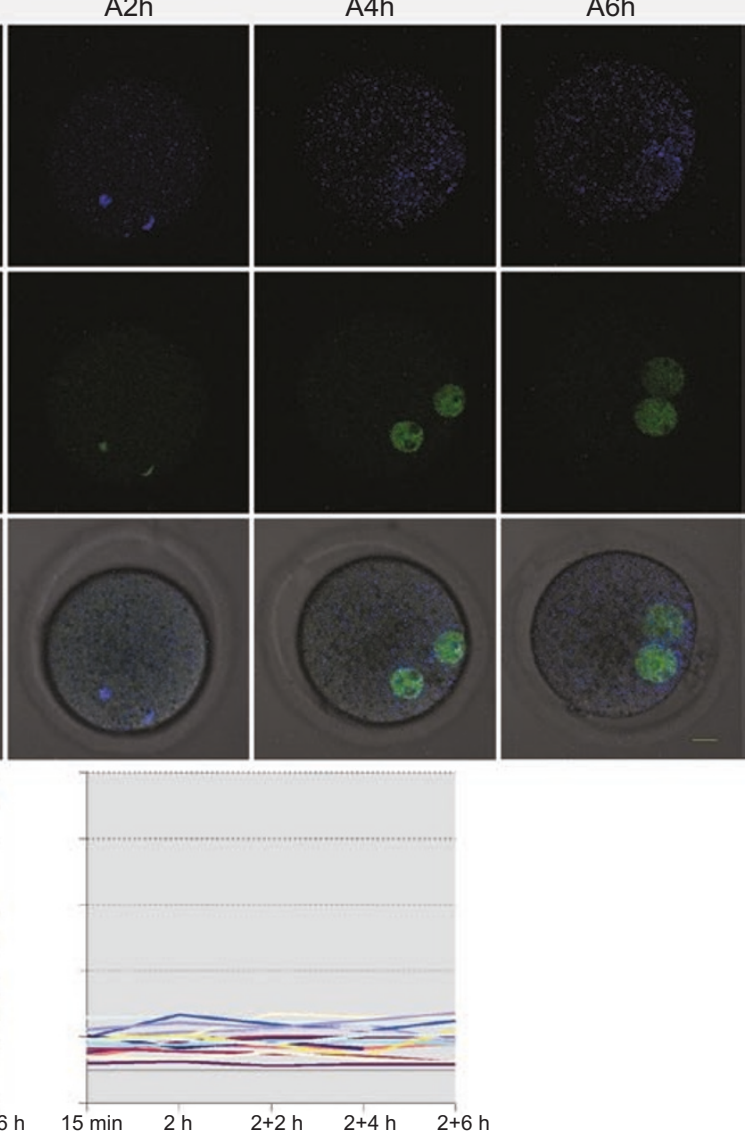

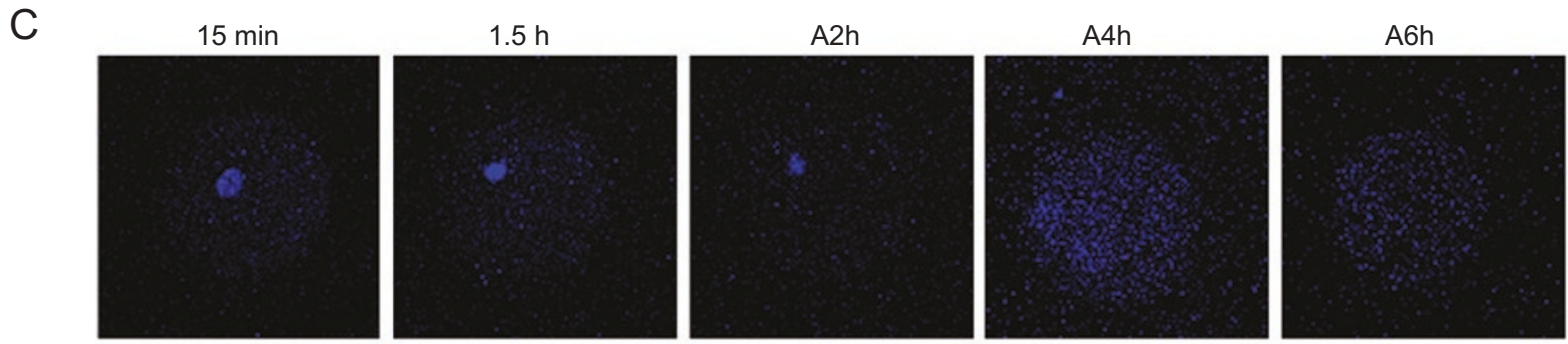

C-1


Figure 3 Subcellular distribution of GFP-CFs in nt-embryos (see also Supplementary information II for controls and additional data). (A) Subcellular distribution of GFP-TBP in nt-embryos. (A-1) Micrographs showing subcellular distribution of GFP-TBP (green) in a reprogrammed nt-embryo. GFP-TBP was in the transplanted nucleus soon after transplantation (15 min), but was no longer visible at $1.5 \mathrm{~h}$ after fusion or 1 and $2 \mathrm{~h}$ after activation $(1.5 \mathrm{~h}, \mathrm{~A} 1 \mathrm{~h}, \mathrm{~A} 2 \mathrm{~h})$. GFP-TBP reappeared in PPNs shortly after their formation (A4h, A6h). Note that Hoechest 33342 (blue) was concentrated in the nucleus at all time points in the failed-to-reprogram embryo (Supplementary information II), but was found on structure outside pronuclei in later stage reprogrammed embryos. 15 min and $1.5 \mathrm{~h}$ indicate times after transplantation. A1h, A2h, A4h, and A6h indicate hours after chemical activation of nt-embryos. Bar $=10 \mu \mathrm{m}$. (A-2) An overview in nuclear concentration of GFP-TBP in reprogrammed (left panel) and failed-to-reprogram groups (right panel), respectively. Each nt-embryo was assigned to one of the three groups: (1) reprogrammed, (2) failed-to-reprogram, and (3) died or showed distorted morphology during the experiment. The third group was discarded. Data from the first and second groups were presented in the diagrams. Each line in these diagrams represented values derived from 1 nt-embryo at five or six different time points. (B) Subcellular distribution of GFP-HP1 $\beta$ in nt-embryos. (B-1) Micrographs showing subcellular distribution of GFP-HP1 $\beta$ (green) in a reprogrammed nt-embryo. It was in the transplanted nucleus at 15 min after fusion, became barely visible at $1.5 \mathrm{~h}$, and was not detected on chromatin at $1 \mathrm{~h}$ after activation (A1h). GFP-HP1 $\beta$ became detectable at $2 \mathrm{~h}$ after activation (A2h) and remained associated with chromatin/nuclei thereafter (A4h and A6h). See (A-1) legend for more details. (B-2). An overview in nuclear concentration of GFP-HP1 $\beta$ in reprogrammed (left panel) and failed-to-reprogram groups (right panel), respectively. See (A-2) legend for more details. (C) Subcellular distribution of GFP-H2B in nt-embryos. (C-1) Micrographs showing subcellular distribution of GFP-H2B (green) in a reprogrammed nt-embryo. GFP-H2B was associated with the nuclei/chromatin throughout the course of nuclear reprogramming. Its intensity appeared to decrease gradually in the reprogrammed nucleus. See (A-1) legend for more details. (C-2) An overview in nuclear concentration of GFP-H2B in reprogrammed (left panel) and failed-to-reprogram groups (right panel), respectively. See (A-2) legend for more details. 
results indicated that the amount of GFP-CFs lost from transplanted nuclei varied drastically from one embryo to another, and only a small percentage $(15 \%)$ of the ntembryos lost $>99 \%$ of GFP-CFs. Re-association between GFP-CF fusion proteins and chromatin/pronuclei began soon after pronuclear formation (Figure 3 and Supplementary information II). From 2 to $6 \mathrm{~h}$ after activation, the amount of GFP-CFs associated with chromatin/pronuclei increased steadily in most embryos (54/60).

It was noticed that when GFP-CFs dissociated from the somatic nuclei, the fluorescent intensity in the cytoplasm was raised slightly and the fluorescence became concentrated in PPNs a few hours later. Redistribution patterns of GFP-CFs suggest that at least a portion of dissociated GFP-CFs is not degraded and those proteins are recruited back to PPNs after their formation. This assumption, while awaiting further experimental proof, is consistent with previous observations. When oocytes or eggs were pre-labeled with isotope and fertilized subsequently, the labeled non-histone proteins in the cytoplasm of the egg migrated into the nuclei of the embryos [36]. Alternatively, when somatic nuclei were transplanted into pre-labeled eggs, isotope-labeled cytoplasmic proteins were incorporated into the transplanted nuclei [13].

In summary, analyses of GFP-coupled TBP, TRF3, TFIIB, BRF 1 , and HP $1 \beta$ in reprogrammed nt-embryos indicate that these GFP-CFs move from somatic nuclei to egg cytoplasm soon after the nuclei contact the cytoplasm of eggs. Dissociation of these GFP-CFs reaches a maximum within a few hours after nt, and before the formation of pronuclei. The amount of GFP-CFs dissociated from somatic nuclei varied dramatically among nt-embryos and is incomplete in most cases. Some of the displaced GFP-CFs may not be degraded and may be later recruited back into pronuclei. Loading of GFP-CFs occurs shortly after pronuclear formation, and nuclear concentrations of these GFP-CFs increase rapidly thereafter. These data matched well with the results from immunochemical analyses for most of the CFs examined. In the case of $\mathrm{MeCP} 2$, it is possible that although some MeCP2 molecules were dissociated from

Table 2 A summary in subcellular distribution of CFs in parthenogenetic embryos

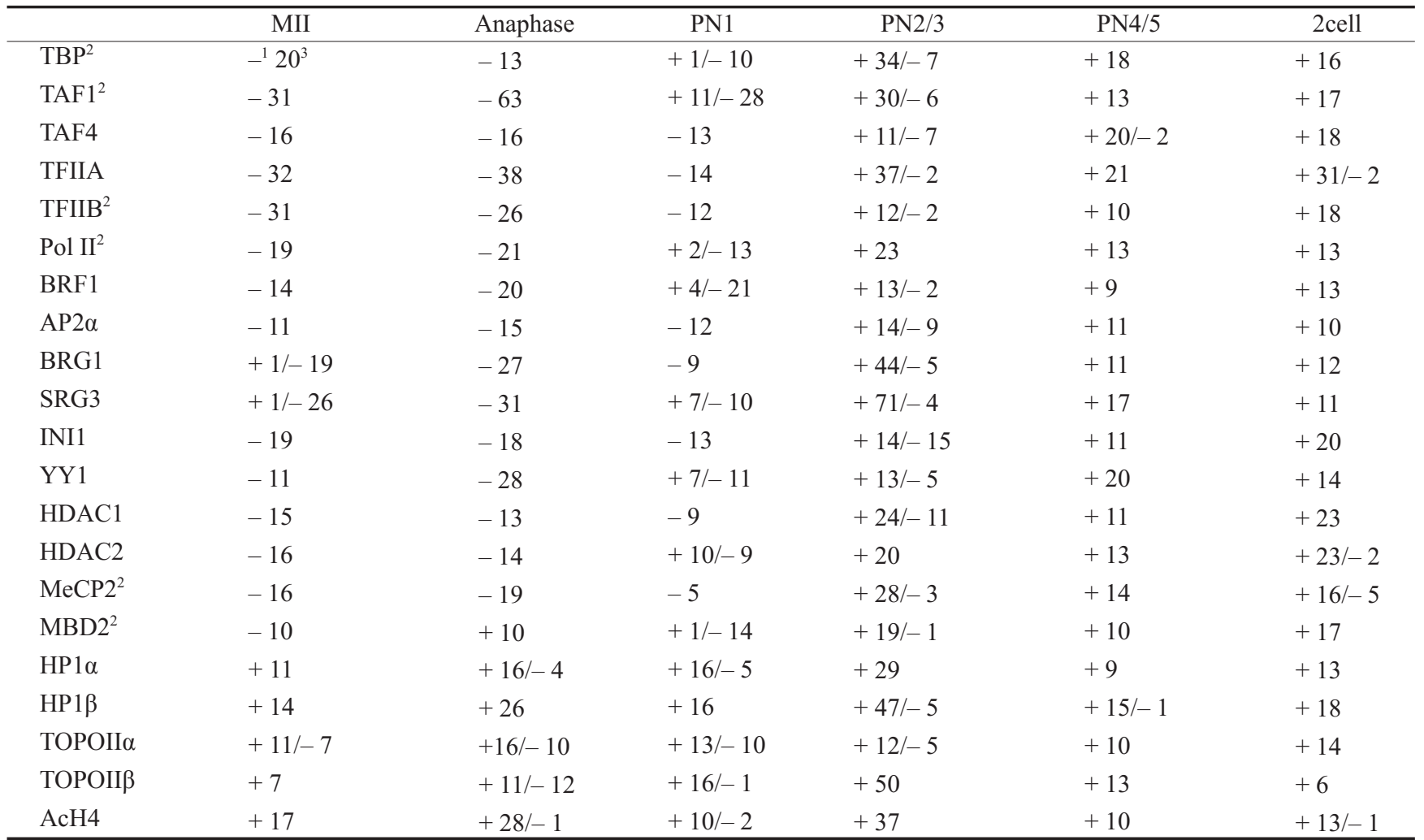

${ }^{1}+$ and - : signal was or was not detected on chromosomes or PNs, respectively.

${ }^{2}$ Signal is also on structures in the vicinity of chromosomes.

${ }^{3}$ The numbers of embryos with the phenotype (all embryos examined are included in the table, i.e., when only one number is in the column, $100 \%$ of the embryos had the particular phenotype). 
A



$\mathrm{MII}^{*}$

Ana
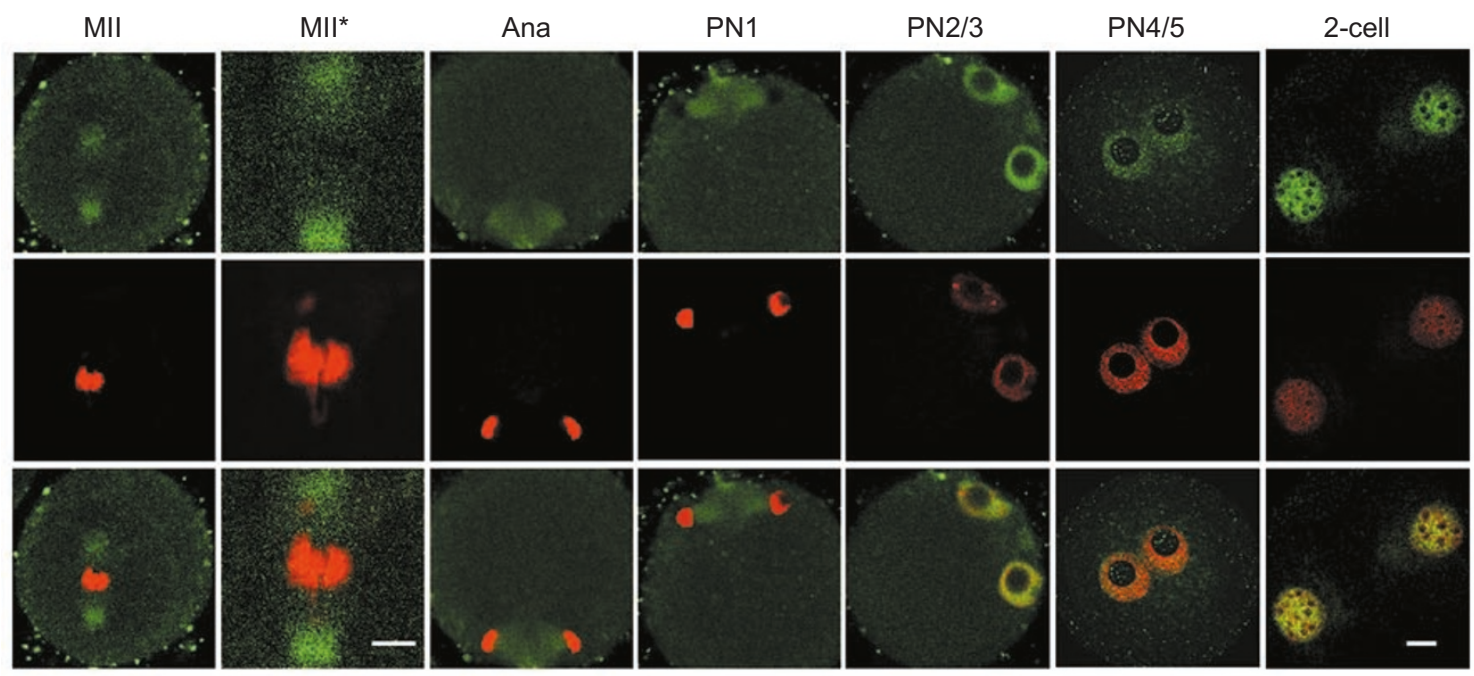

N2/3

PN4/5

2-cell

B

MII

Ana

PN1

PN2/3

PN4/5

2-cell
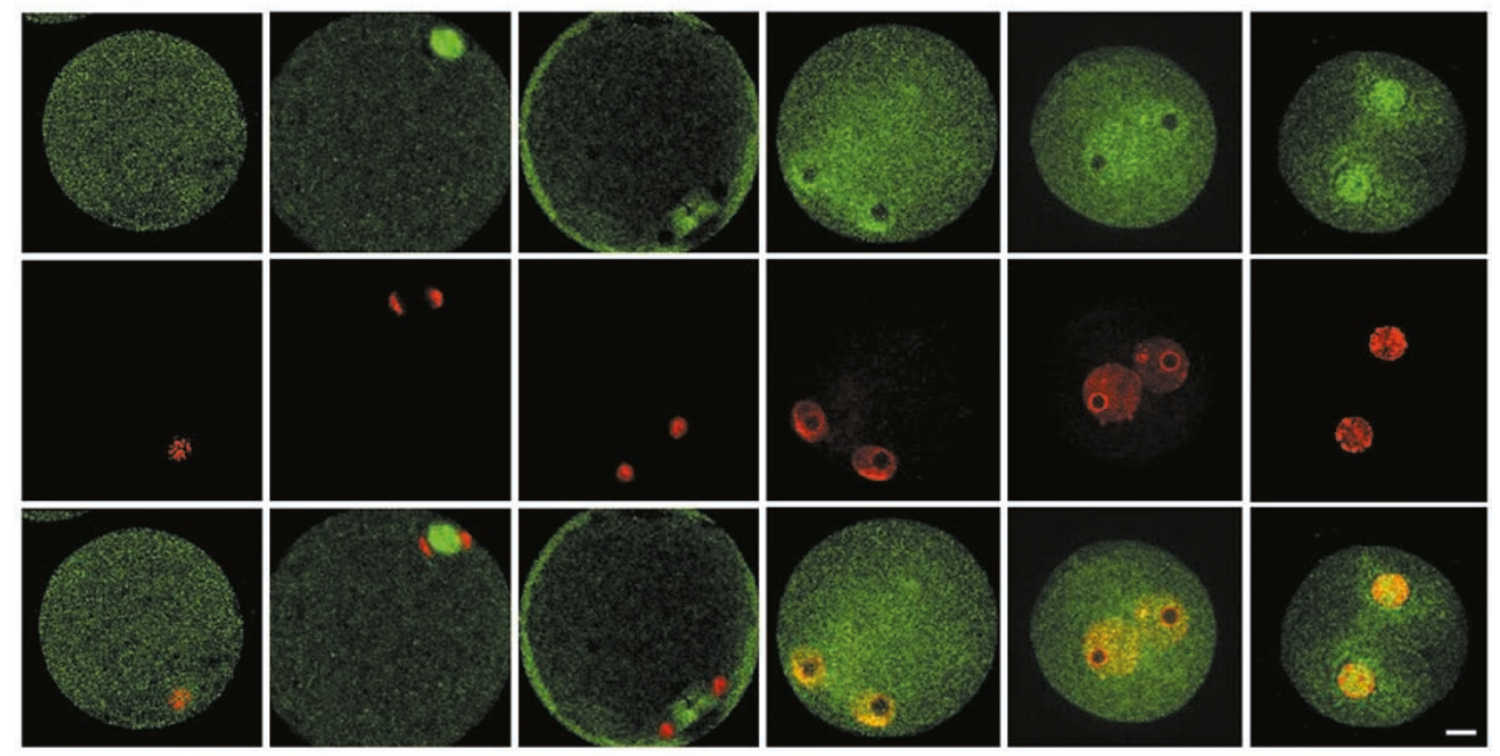

C

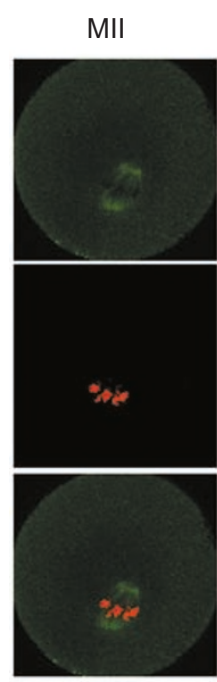

MII*

PN1

PN2/3

PN4/5

2-cell
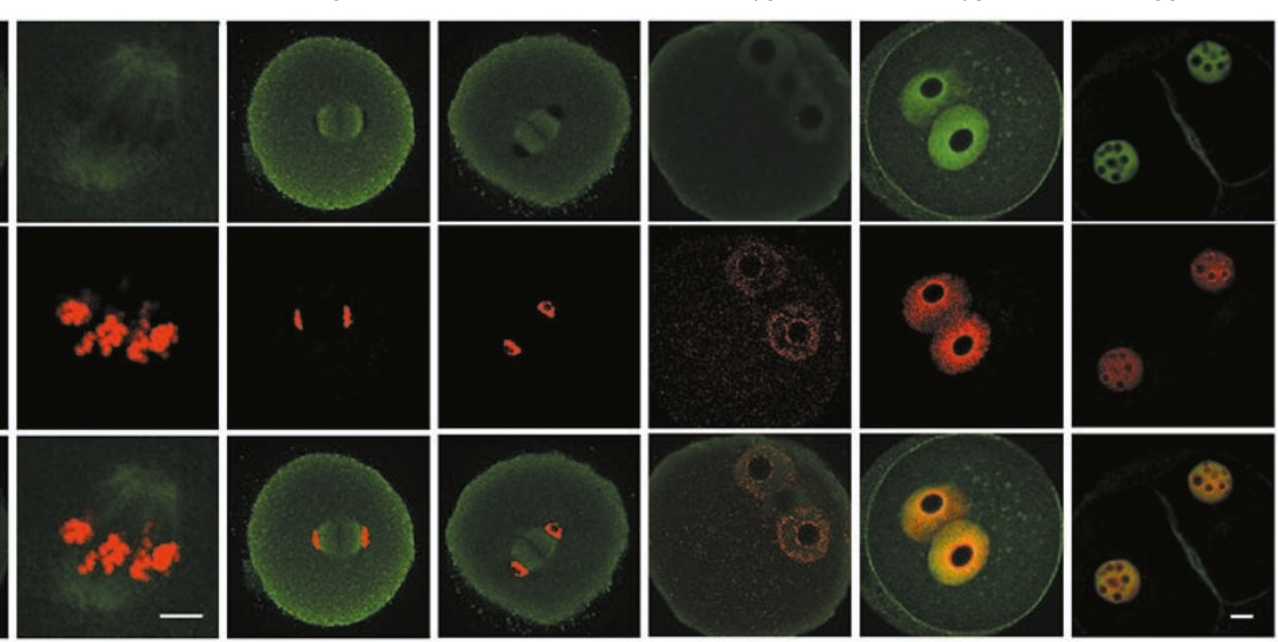


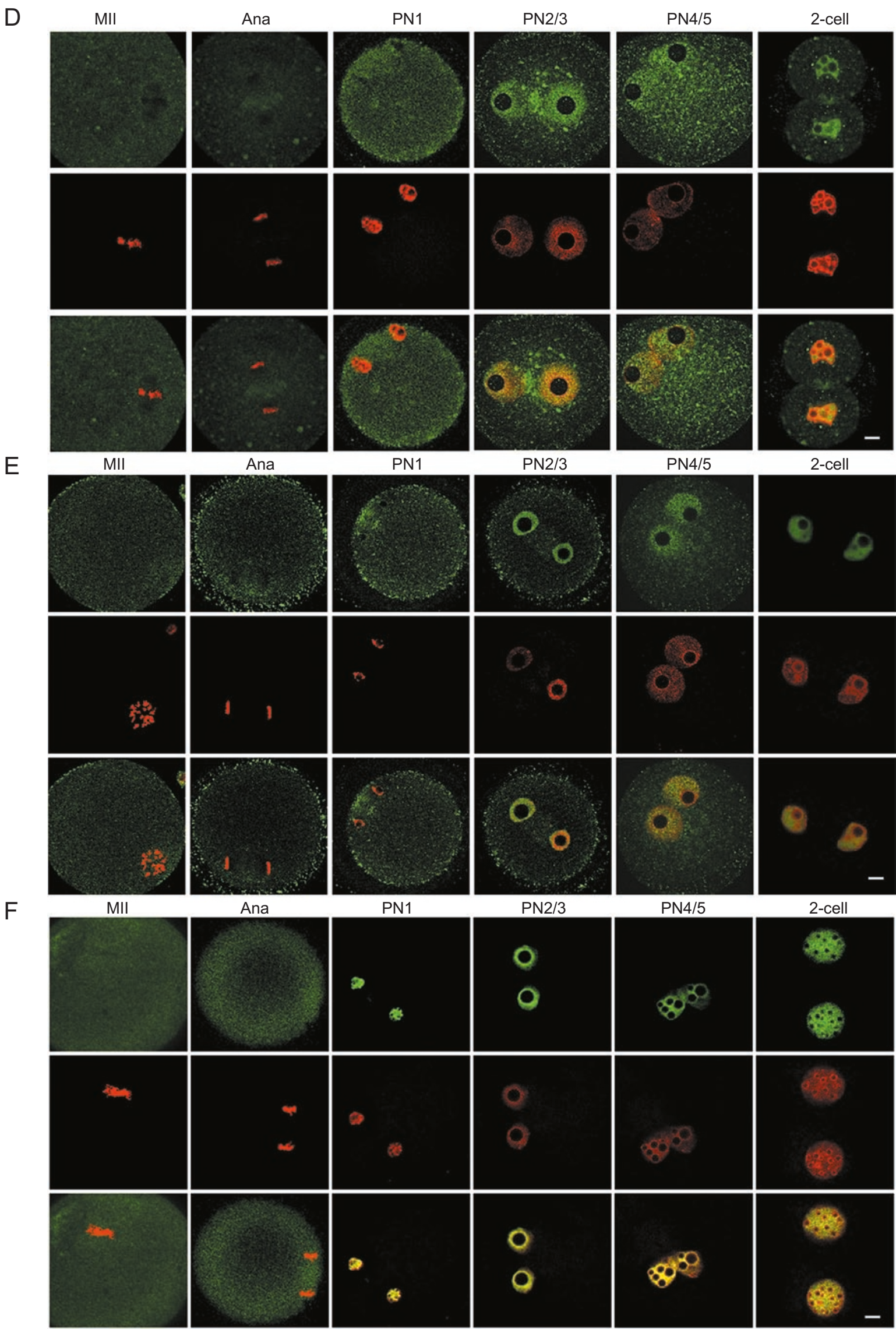




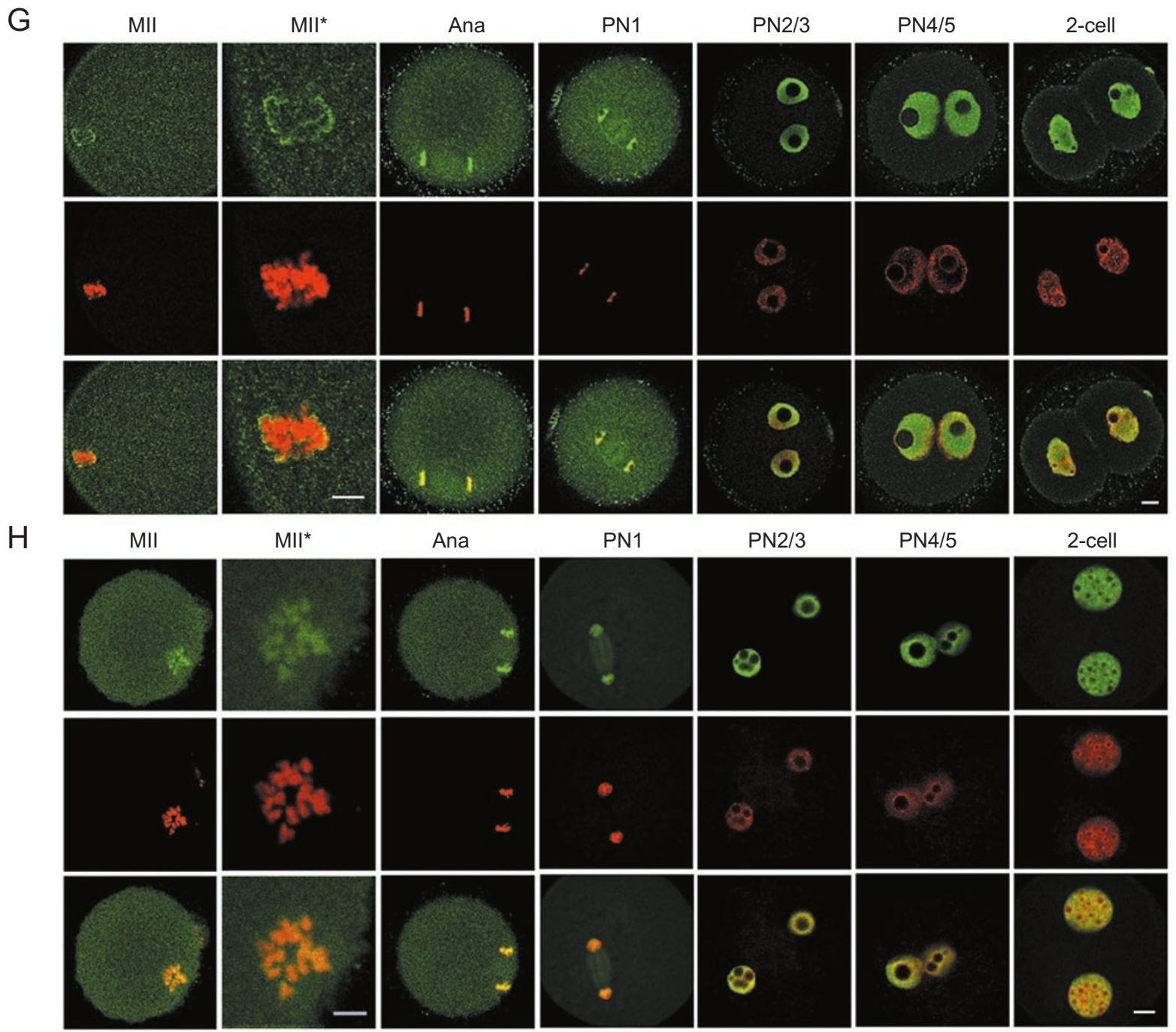

Figure 4 Dynamic changes in nucleus-CF relationship in parthenogenetic embryos as revealed by indirect immunofluorescent assays. TBP (A), TAF1 (B), TFIIB (C), BRG1 (D), SRG3 (E), and HDAC2 (F) (green), were not detected on chromosomes (red) before parthenogenetic activation (MII), and became detected in nuclei/chromatin shortly after pronuclear formation (Ana, PN1, PN3/4, PN4/5, 2-cell). The HP1 $\beta$ signal (G) was on but was not completely overlaid with chromosome in some MII eggs, and it was detected on chromosomes/nuclei in all other stages. AcH4 (H) was on chromatin/nuclei throughout the course. * denotes a high magnification image of the left. Bar $=10$ and $5 \mu \mathrm{m}$ in low and high magnitude, respectively. See Supplementary information III for more details with these CFs.

chromatin, a low amount of them was retained, which is below the detection threshold of the antibody (see Sun F, Sun F, et al., Cell Res 2007; Supplementary information II-7 for more discussion).

\section{Reloading of CFs to the pre-erased chromatin in parthe- nogenotes}

Parthenogenotes have undergone normal oogenesis, and should have regenerated a set of relatively naïve chromatin. This was confirmed by antibody-staining results: except for
HP1 $\alpha$, HP $1 \beta$, TOPOII $\alpha$, TOPOII $\beta$ and AcH4, the other 16 CFs were not detected on chromosomes in MII eggs before parthenogenesis was initiated (Figure 4, Supplementary information III, and Table 2). According to the "erase-andrebuild" model, dissociated CFs must be loaded back to chromatin before development takes place. To test this and to analyze the dynamics of the loading process, parthenogenotes at early developmental stages were examined. TBP, TAF1, TAF4, TFIIA, TFIIB, BRF1, Pol II, AP2 $\alpha$, BRG1, SRG3, INI1, YY1, MeCP2, MBD2, HDAC1, and HDAC2 
remained undetectable on chromosomes in anaphase. Two female pronuclei (FPNs) formed approximately 2 to $3 \mathrm{~h}$ after activation. The $16 \mathrm{CFs}$ became positive in FPNs soon after their formation. At $12 \mathrm{~h}$ after activation, a time when transcription is expected to take place, all CFs were detected in the nuclei and persisted there from then on (Figure 4, Supplementary information III, and Table 2). Data from these experiments demonstrate that the CFs were loaded back to chromatin in a temporal order very similar to that in normal development.

\section{Discussion}

As demonstrated in the accompanying paper [41] that an "erase-and-rebuild" strategy is used in normal development to reset the maternal transcriptional profile to that of the embryo, we show in this paper that during SCNT and parthenogenesis, CFs critical for transcription activation and regulation are dissociated from somatic nuclei and duplicated maternal chromatin, respectively, in manners analogous to the erasure process in oogenesis. Most CFs later become re-associated with pronuclei/chromatin during SCNT and parthenogenesis in a spatiotemporal manner similar to that in fertilized embryos. These data indicate that the "erase-and-rebuild" strategy is also used to establish the zygotic transcription program during SCNT and parthenogenesis, albeit in altered fashions.

Protein exchanges between transplanted nuclei and the cytoplasm of oocytes/eggs had been described many decades ago: "uptake and release of proteins into and from transplanted nuclei are roughly proportional to nuclear enlargement and do not take place in nuclei which fail to enlarge and which would not support normal development". "It appears that nuclear swelling, chromatin dispersal, and protein exchange may be causally connected with reprogramming" $[4,13]$.

Data from present studies agree with previous analyses and develop further along the same direction. We have reached a number of conclusions: (1) Somatic cell reprogramming is composed of two consecutive phases. In the first phase, soon after nt, a broad range of CFs moves out of the somatic nucleus. In the second phase, shortly after PPN formation, most CFs are loaded back to chromatin. (2) Dissociation of most CFs from chromatin disrupts contacts between chromatin and protein complexes. It erases the existing transcription program in somatic nuclei. Subsequently, re-association between chromatin and CFs in PPNs makes it possible to establish a new transcription program. (3) Protein exchange between transplanted nuclei and the cytoplasm always follows the same order: CFs move from chromatin/nucleus to cytoplasm and then enter the pronuclei. This order is conserved in all three reprogramming processes underlying normal development, SCNT, and parthenogenesis. It has an apparent functional implication - the existing transcription program, and most likely other epigenetic information as well, needs to be cleared off the chromatin before a new program can be installed appropriately. (4) The identity of proteins being exchanged is now clear. There is not a single or a few factors involved, but an extensive range of CFs. This conclusion is based on the fact that a majority of CFs $(15 / 20)$ examined in this study have undergone a dissociation and re-association process with nuclei during SCNT. This conclusion is also supported by previous observations that somatic nuclei lose a large proportion (approximately 80-90\%) of nonhistone proteins after transplantation $[4,13]$ or contacting egg extract [35].

Our data imply that GV nuclei contain activities promoting $\mathrm{CF}$ dissociation, which appeared to be released to the cytoplasm after GVBD, and kept active for a prolonged time. Such activities are able to cause global CF dissociation from somatic nuclei during SCNT. The CF dissociation (the erasure) process can be fundamental for "de-differentiation" of somatic cells. It may function through either removing repressive mechanisms from the differentiated cell and/or creating a relative naïve set of chromatin onto which the zygotic program will be built.

Compared with normal development, there is only a narrow window during SCNT (from the time when the somatic cell is fused to the egg to the time when pronuclei form) to remove the existing epigenetic program from chromatin. In addition, the environment and strength of the activities promoting $\mathrm{CF}$ dissociation in egg may have changed from that in GV oocytes. The GFP-CF fusion protein experiment showed that dissociation of CFs from somatic nuclei/chromatin was extremely variable among nt-embryos, and in most cases was incomplete. Similar observations were recorded in previous publications [37]. A less-than complete dissociation of CFs from chromatin, and an incomplete erasure of other epigenetic marks [712], will result in continued expression of the existing transcription program. It explains the observation that a substantial proportion of nt-embryos retains a variable amount of ectopic gene expression and aberrant epigenetic memory related to the developmental history of the nuclear donors [7-12, 15], and the observation that after embryo reconstruction a prolonged pre-incubation time, which presumably facilitates $\mathrm{CF}$ dissociation and possibly removal of other epigenetic marks, often leads to better reprogramming [30, 31]. Incomplete erasure of the somatic transcription program can interfere with installation and execution of zygotic developmental program. This can be causally related to the high percentage of developmental failure seen in SCNT. Approaches directed at enhancing 
erasure of the existing epigenetic program, including the transcription program, in somatic nuclei may improve success rates in SCNT.

The global dissociation and re-association of CFs from chromatin occur in parallel with other reprogramming processes, e.g. DNA demethylation [38] and histone deacetylation [29, 39] during SCNT. The combinatorial effect of these processes resets the somatic nuclei to that of the embryo. In addition, the "erase-and-rebuild" process should reprogram not only transcription but also other nuclear processes, e.g. DNA replication [40].

Shortly after pronuclear formation, most CFs are loaded back to nuclei. The timing and dynamics of loading are similar in normal development, SCNT and pathernogenesis, and embryos resulting from all three reproductive procedures develop along the same path. This implies that eggs have installed the same developmental program to the nuclei/chromatin of different origins, and that the zygotic developmental program is the only program that eggs install. The nature of the developmental program to be installed is thus determined by eggs, not by the chromatin. Furthermore, parthenogenotes initiate and retrace the normal developmental path in the absence of any addition(s) to the egg, e.g. a sperm or somatic nucleus. This indicates that the egg contains most, if not all, essential components for assembling the zygotic developmental program, including the transcription program.

According to the "erase-and-rebuild" model, the existing transcription program in the diploid chromatin will be erased anyway; the developmental history of the nucleus (e.g. a somatic nucleus of a particular cell type or differentiation stage), therefore, should not influence the installation and execution of the zygotic program. This assumption has been demonstrated to be true experimentally [17]. Since eggs install only the zygotic program, embryos resulting from SCNT and parthenogenesis should retrace always the same zygotic developmental path. Based on the fact that SCNT and parthenogenesis use the reprogramming mechanism of normal development to establish new life cycles, they can be regarded as variations of normal development, although retention of somatic epigenetic memory in nt-embryos [15] and an imbalanced imprinting pattern in parthenogenotes [26] will lead to unique developmental consequences, respectively.

\section{Acknowledgments}

We are grateful to Dr Yun-Bo Shi, Yingzi Yang, and Paul Zhou for criticizing the manuscript. We are thankful to Dr Shangang Li for assistance in nuclear transfer procedure, Wei Su for artwork, Youming Zhu for cell culture, Wei Liu for assistance in immunochemistry, and Fengying Li, Wen- qin Ying, and Wanli Li for animal care. We also apologize for citing reviews instead of original publications in some places due to space limitations. Please look in the reviews for original papers.

The study was supported by grants from National Basic Research Program of China (973 Program) (No. 001CB509903, 001CB509904), Hi-Tech Research and Development Program of China (863 Program) (No. 2001AA216121, 2004AA205010), Science and Technology Committee of Shanghai Municipality (No. 99DJ14002, 00DJ1 4033, 01DJ14003, 03DJ14017), Shanghai Municipal Education Commission (No. T0205) and Shanghai Jiao Tong University, School of Medicine.

Contributions: Tianlong Gao and Haiyan Fang are responsible for GFP-CF analyses, Junke Zheng, Feng Sun, Ayong Yan, Xun Gong, Hui Ding, and Fang Tang for analyses on nt-embryos, Fengying Xing for analyses in parthenogenotes. Feng Sun assisted in manuscript writing. Hui Z Sheng is responsible for development of the model, project planning, and most manuscript writing.

\section{References}

1 Meissner A, Jaenisch R. Mammalian nuclear transfer. Dev Dyn 2006; 235:2460-2469.

2 Collas P, Pinto-Correia C, Ponce de Leon FA, Robl JM. Effect of donor cell cycle stage on chromatin and spindle morphology in nuclear transplant rabbit embryos. Biol Reprod 1992; 46:501511.

3 Campbell KH, Ritchie WA, Wilmut I. Nuclear-cytoplasmic interactions during the first cell cycle of nuclear transfer reconstructed bovine embryos: implications for deoxyribonucleic acid replication and development. Biol Reprod 1993; 49:933-942.

4 Gurdon JB, Laskey RA, De Robertis EM, Partington GA. Reprogramming of transplanted nuclei in amphibia. Int Rev Cytol Suppl 1979:161-178.

5 Eggan K, Akutsu H, Hochedlinger K, Rideout W III, Yanagimachi $\mathrm{R}$, Jaenisch R. X-Chromosome inactivation in cloned mouse embryos. Science 2000; 290:1578-1581.

6 Lanza RP, Cibelli JB, Blackwell C, et al. Extension of cell life-span and telomere length in animals cloned from senescent somatic cells. Science 2000; 288:665-669.

7 Dean W, Santos F, Stojkovic M, et al. Conservation of methylation reprogramming in mammalian development: aberrant reprogramming in cloned embryos. Proc Natl Acad Sci USA 2001; 98:13734-13738.

8 Kang YK, Koo DB, Park JS, et al. Aberrant methylation of donor genome in cloned bovine embryos. Nat Genet 2001; 28:173177.

9 Humpherys D, Eggan K, Akutsu H, et al. Epigenetic instability in ES cells and cloned mice. Science 2001; 293:95-97.

10 Bourc'his D, Le Bourhis D, Patin D, et al. Delayed and incomplete reprogramming of chromosome methylation patterns in bovine cloned embryos. Curr Biol 2001; 11:1542-1546.

11 Inoue K, Kohda T, Lee J, et al. Faithful expression of imprinted genes in cloned mice. Science 2002; 295:297. 
12 Kang YK, Park JS, Koo DB, et al. Limited demethylation leaves mosaic-type methylation states in cloned bovine pre-implantation embryos. EMBO J 2002; 21:1092-1100.

13 Gurdon JB. Nuclear transplantation in eggs and oocytes. J Cell Sci Suppl 1986; 4:287-318.

14 Kim JM, Ogura A, Nagata M, Aoki F. Analysis of the mechanism for chromatin remodeling in embryos reconstructed by somatic nuclear transfer. Biol Reprod 2002; 67:760-766.

$15 \mathrm{Ng}$ RK, Gurdon JB. Epigenetic memory of active gene transcription is inherited through somatic cell nuclear transfer. Proc Natl Acad Sci USA 2005; 102:1957-1962.

16 Surani MA. Nuclear reprogramming by human embryonic stem cells. Cell 2005; 122:653-654.

17 Hochedlinger K, Jaenisch R. Nuclear reprogramming and pluripotency. Nature 2006; 441:1061-1067.

18 Gurdon JB, Byrne JA. The first half-century of nuclear transplantation. Biosci Rep 2004; 24:545-557.

19 Vrana KE, Hipp JD, Goss AM, et al. Nonhuman primate parthenogenetic stem cells. Proc Natl Acad Sci USA 2003; 100 Suppl 1:11911-11916.

20 Surani MA, Barton SC. Development of gynogenetic eggs in the mouse: implications for parthenogenetic embryos. Science 1983; 222:1034-1036.

21 Surani MA, Kothary R, Allen ND, et al. Genome imprinting and development in the mouse. Dev Suppl 1990; 89-98.

22 McGrath J, Solter D. Completion of mouse embryogenesis requires both the maternal and paternal genomes. Cell 1984; 37:179-183.

23 Kure-bayashi S, Miyake M, Okada K, Kato S. Successful implantation of in vitro-matured, electro-activated oocytes in the pig. Theriogenology 2000; 53:1105-1119.

24 Hagemann LJ, Peterson AJ, Weilert LL, Lee RS, Tervit HR. In vitro and early in vivo development of sheep gynogenones and putative androgenones. Mol Reprod Dev 1998; 50:154-162.

25 Barton SC, Surani MA, Norris ML. Role of paternal and maternal genomes in mouse development. Nature 1984; 311:374-376.

26 Kono T. Genomic imprinting is a barrier to parthenogenesis in mammals. Cytogenet Genome Res 2006; 113:31-35.

27 Kono T, Obata Y, Wu Q, et al. Birth of parthenogenetic mice that can develop to adulthood. Nature 2004; 428:860-864.

28 Chatot CL, Ziomek CA, Bavister BD, Lewis JL, Torres I. An improved culture medium supports development of random-bred 1-cell mouse embryos in vitro. J Reprod Fertil 1989; 86:679688.

29 Adenot PG, Mercier Y, Renard JP, Thompson EM. Differential $\mathrm{H} 4$ acetylation of paternal and maternal chromatin precedes DNA replication and differential transcriptional activity in pronuclei of 1-cell mouse embryos. Development 1997; 124:4615-4625.

30 Wakayama T, Perry AC, Zuccotti M, Johnson KR, Yanagimachi R. Full-term development of mice from enucleated oocytes injected with cumulus cell nuclei. Nature 1998; 394:369-374.

31 Inoue K, Ogonuki N, Mochida K, et al. Effects of donor cell type and genotype on the efficiency of mouse somatic cell cloning. Biol Reprod 2003; 69:1394-1400.

32 Worrad DM, Ram PT, Schultz RM. Regulation of gene expression in the mouse oocyte and early preimplantation embryo: developmental changes in Sp1 and TATA box-binding protein, TBP. Development 1994; 120:2347-2357.

33 Wang K, Sun F, Sheng HZ. Regulated expression of TAF1 in 1-cell mouse embryos. Zygote 2006; 14:209-215.

34 Sun F, Tang F, Yan AY, Fang HY, Sheng HZ. Expression of SRG3, a chromatin remodeling factor, in the mouse oocyte and early preimplantation embryos. Zygote 2007; 15:1-10.

35 Kikyo N, Wade PA, Guschin D, Ge H, Wolffe AP. Active remodeling of somatic nuclei in egg cytoplasm by the nucleosomal ATPase ISWI. Science 2000; 289:2360-2362.

36 Ecker RE, Smith LD. The nature and fate of Rana pipiens proteins synthesized during maturation and early cleavage. Dev Biol 1971; 24:559-576.

37 Gurdon JB, De Robertis EM, Partington G. Injected nuclei in frog oocytes provide a living cell system for the study of transcriptional control. Nature 1976; 260:116-120.

38 Simonsson S, Gurdon J. DNA demethylation is necessary for the epigenetic reprogramming of somatic cell nuclei. Nat Cell Biol 2004; 6:984-990.

39 Kim JM, Liu H, Tazaki M, Nagata M, Aoki F. Changes in histone acetylation during mouse oocyte meiosis. J Cell Biol 2003; 162:37-46.

40 Lemaitre JM, Danis E, Pasero P, Vassetzky Y, Mechali M. Mitotic remodeling of the replicon and chromosome structure. Cell 2005; 123:787-801.

41 Sun F, Fang H, Li R, et al. Nuclear reprogramming: the zygotic transcription program is established through an "erase-and-rebuild" strategy. Cell Res 2007; 17:117-134.

(Supplementary Information is linked to the online version of the paper on the Cell Research website.) 